\title{
Calendrier des manifestations
}

19 janvier 2019, Zurich

Formation continue : Éthique, code déontologique et obligations en psychothérapie Intervernante : Marianne Meister-Notter Organisateur : ASP

Info : www.psychotherapie.ch

20 janvier 2019, Zurich

Formation post-graduée/formation continue : Droits et obligations dans la relation à la patiente, au patient - un aperçu

Intervernante : Cornelia Kranich Schneiter (avec Peter Schulthess)

Organisateur : ASP

Info : www.psychotherapie.ch

23 mars 2019, Zurich

Assemblée des membres ASP

(40e anniversaire)

Organisateur : ASP

Info : www.psychotherapie.ch

6 avril 2019, Zurich

Formation post-graduée/

formation continue : Approches

et méthodes dans la psychothérapie :

Approches systématiques

Intervenant : Martin Rufer

Organisateur : ASP

Info : www.psychotherapie.ch

7 avril 2019, Zurich

Formation post-graduée/

formation continue : Approches

et méthodes dans la psychothérapie :

Procédé humaniste et intégratif

Intervenant : Peter Schulthess

Organisateur : ASP

Info : www.psychotherapie.ch

3 - 7 juillet 2019, Buenos Aires, Argentine $50 \mathrm{e}$ rencontre annuelle internationale de la SPR

Organisateur : SPR

Info : https://www.psychotherapyresearch. org/events/event_list.asp
4 - 6 septembre 2019, Berne

Congrès annuel de la SSPP et SGKJP

Info : www.psy-congress.ch

7 - 8 septembre 2019, Zurich

Formation post-graduée/

formation continue : Recherche

en psychothérapie et ses implications

pour la pratique

Intervenant : Volker Tschuschke

Organisateur : ASP

Info : www.psychotherapie.ch

19 - 21 septembre 2019, Cracovie, Pologne 5 e rencontre commune $R$. $U$.

et Européenne de la SPR

Organisateur: SPR

Info : https://www.psychotherapyresearch.

org/events/event_list.asp

16 - 17 novembre 2019, Zurich

Formation post-graduée/

formation continue :

Interrogations éthiques

et contextes sociopolitiques

Intervenant : Alberto Bondolfi

Organisateur : ASP

Info : www.psychotherapie.ch

17 - 20 juin 2020, Amherst, USA

51e rencontre annuelle internationale

de la SPR

Organisateur : SPR

Info : https://www.psychotherapyresearch.

org/events/event_list.asp

26 - 29 juin 2020, Moscou, Russie $9^{\text {th }}$ World Congress for Psychotherapy

Organisateur : WCP

(World Council for Psychotherapy)

Info : www.planetofpsychotherapy.com 